\title{
Counterion Condensation of a Polyelectrolyte within a Micro/Nanochannel
}

\author{
Cheng-Hsuan Huang, Yu-Fan Lee, and Eric Lee
}

\begin{abstract}
Counterion condensation phenomenon of a highly charged polyelectrolyte is directly related to DNA (DeoxyriboNucleic Acid) condensation and RNA (RiboNucleic Acid) folding, which are research topics of vital interest in areas like biological and medical sciences as well as electrokinetic separations. With the advances of the modern micro/nanofabrication technology, practical applications are normally conducted in corresponding micro/nanochannels. As a result, a fundamental understanding of the counterion condensation phenomenon within a micro/nanochannel is crucial for successful operations utilizing it. We provide a theoretical analysis here with the polyelectrolye modelled as a charged porous sphere, and the full nonlinear Poisson equation is adopted to describe the interaction between the counterions in the suspending electrolyte solution and the backbone macroion of the polyelectrolyte itself. The solution is obtained numerically with a pseudo-spectral method based on Chebyshev polynomials. The degree of counterion condensation is expressed as a function of various parameters of interest, such as the charge condition of the polyelectrolyte, the ratio of the particle to pore radii, and the ionic strength of the suspending electrolyte solution. Results presented here provide useful information relevant in various practical applications such as biosensors, DNA stretching, and gene delivery.
\end{abstract}

Index Terms-Boundary confinement effect, counterion condensation, nanostructure, polyelectrolyte.

\section{INTRODUCTION}

"Polyelectrolyte" is a term first introduced by Fuoss [1] to describe a substance of high molecular weight which is simultaneously an electrolyte. It is a macromolecule bearing a number of dissociable functional groups which can dissociate into charged polymer chains (macroions) and small counter-ions. Many organic and biological entities are polyelectrolytes, such as proteins, polysaccharides, DNA, and so on. Synthetic polymers in industry such as PAA (polyacrylic acid), PMA (polymethacrylic acid), and PAMAM (polyamidoamine) are polyelectrolytes as well [2]. The broad spectrum of variability of polyelectrolytes opens a lot of practical applications, for example, gene delivery has been accomplished using a variety of positively charged polyelectrolyte to form DNA complexes and transfect cultured cells with lower toxicities and higher efficiencies [3].

Manuscript received June 12, 2015; revised August 22, 2015. This work is partially supported by the Ministry of Science and Technology, Republic of China.

C. H. Huang was with the Department of Chemical Engineering, National Taiwan University, Taipei, Taiwan. He is now with Taiwan Semiconductor Manufacturing Company (e-mail: hsuanhzb@tsmc.com).

Y. F. Lee and E. Lee are with the Department of Chemical Engineering, National Taiwan University, Taipei, Taiwan (e-mail r03524038@ntu.edu.tw, ericlee@ntu.edu.tw).
It also found many novel applications in different fields, such as drag delivery, phase separation in pharmacy and cosmetics, cell fixation in biotechnology, and the fabrication of soft materials in nanotechnology [4], [5]. Because of their fundamental importance in biology and biochemistry, polyelectrolytes have been the object of a continued interest since the early days of polymer science.

Electrostatic behavior is a key factor when investigating of a charged polyelectrolyte and that is fundamentally different from the conventional small particles. When the ions concentration in the bulk solution increases, the double layer surrounding the particle is suppressed and more counterions are driven into the interior of the porous particle. This phenomenon is referred to as the "shielding effect" by some researchers [6], as the strong Coulomb repulsive interaction between fixed charges is somehow "shielded" by the existence of counterions in between. Note that this shielding effect leads to the well-known counterion condensation, where the thermal energy of the counterions is insufficient for them to escape the electric field generated by the large number of charges on the macroion backbone [7]. The occurrence of shielding effect leads to a neutralization of the total charge of macroions, which is directly related to some important phenomena in biology, such as DNA condensation [8] and RNA folding [9]. DNA condensation, defined as the packing of random coiled DNA into small ordered structures, has been studied extensively. According to Zimm and Le Bret [10], counterion condensation is a phenomenon which can be solidly accounted for based on the Poisson-Boltzmann equation, so the shielding effect here can be viewed as a conceptual extension of the physical condensation of counterions. Hsu and Lee [11] studied a uniformly distributed charged polyelectrolyte by Poisson-Boltzmann equation, and their result successfully described the observed counterion condensation for a giant folded DNA, which cannot be interpreted by the famous theory proposed by Manning [12]. Yeh and Hsu [13] further proposed a $\mathrm{pH}$-regulated model to show the ions $\left(\mathrm{H}^{+}\right.$and $\left.\mathrm{OH}^{-}\right)$dissociated by water have significant influence on the effective charge of a polyelectrolyte.

Thanks to the advances of the micro-/nanofabrication technology, microdevices with even smaller features can be produced now and the electrokinetic technique can be further downscaled to tens or hundreds of nanometers, allowing manipulation of even smaller biological particles, such as virus, bacteria, DNA, chromosomes, and other macromolecules [14]. Similar phenomena are observed in synthetic hydrogels or polymeric ultrafiltration membrane, where the porous membrane can be viewed as the numerous tiny micro/nanopores [15]. For example, the DNA sequencing 
technique utilize gel-electrophoresis very often, and Ma and Bloomfield [16] used agarose gel electrophoresis to measure the effective charge neutralization of DNA by counterions of different structure and valence, including $\mathrm{Na}^{+}, \mathrm{Mg}^{2+}$, and $\mathrm{Co}\left(\mathrm{NH}_{3}\right)_{6}{ }^{3+}$. In their measurements demonstrate that gel electrophoresis is a simple and surprisingly effective method to study the relative binding of counterions to DNA. Moreover, DNA mapping approach with DNA stretching technique has also gained much attention due to its low cost and efficiency [17]-[19]. Among this technique, the screening effect of Coulomb interaction is a crucial factor of the elongation of DNA, which has been explained in terms of self-avoidance. Therefore, it is important to consider aforementioned electrostatic phenomena to develop a comprehensive model of polyelectrolytes in micro/nanochannels. A series of researches results regarding colloidal particle conducting electrophoretic motion within a cylindrical channel has been reported in the literature [20], [21].

To investigate the electrical properties of a polyelectrolyte, it has been common to treat a polyelectrolyte macromolecule as a charged colloid, and Overbeek [22] indicated there are three possible ways to model a polyelectrolyte. Among them, both the coiled cylinder and the porous sphere models have been adopted by various research groups with success in different situations. In reality, according to a recent experimental study [23] on DNA electrophoresis, the actual image of a DNA molecule in free solution, obtained via an optical microscopy, is of a 3-D blob conformation closely resembling a porous sphere. Moreover, as indicated by Salieb-Beugelaar et al. [14], a DNA molecule do not have to deform when it enters a nanostructure of the dimensions larger than twice the radius of gyration, where the nanostructure can be a capillary, nanochannel, nanoslit or a pore matrix (gel). In addition, experimental observation of some specific protein particles by optical microscope demonstrates a porous 3-D blob shape as well, reported by Bell and Dunnill [24]. As a result, it is appropriate to treat the general polyelectrolytes in an electrolyte solution as charged porous spheres, say, 3-D blob shape.

In this study, we investigated the counterion condensation phenomenon of a polyelectrolyte within a micro/nanochannel by solving the full nonlinear Poisson-Boltzmann equation, and the polyelectrolyte modeled as a charged porous sphere with a uniformly distributed constant fixed charge density. In microfluidic operations involving polyelectrolytes such as proteins, the boundary effect becomes important, so we focus on the impact on the effective charge density due to the presence of the micro/nanochannel. Parameters of electrokinetic interest are examined, such as the double layer thickness of the particle, the electric characteristic properties of the porous particle, the ratio of pore-to-particle radii, and so on. A pseudo-spectral method [25] based on Chebyshev polynomials is used to solve the resulting general electrokinetic equations numerically. This study provides important fundamental information about the counterion condensation or the shielding effect of charged porous sphere like polyelectrolytes in micro- and nanochannels, which is essential to the successful designs and operations like biosensors, LOC devices and DNA stretching channels frequently utilized in biochemical, biological, and other fields involving soft matters such as colloids and bio-related materials.

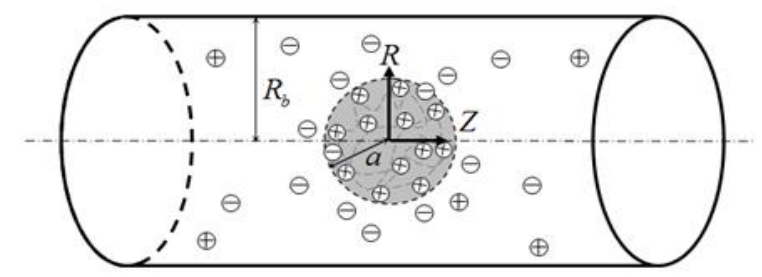

Fig. 1. Schematic representation of the problem considered where a porous particle of radius $a$ is placed on the axis of a long cylindrical pore of radius $R_{b}$.

\section{THEORY}

\section{A. Full Nonlinear Poisson Boltzmann Equation}

As illustrated in Fig. 1, we consider a porous spherical particle of radius $a$ with fixed volumetric charge density $\rho_{f i x}$ in the center of a cylindrical channel of radius $R_{b}$. The cylindrical channel is filled with an aqueous solution containing $z_{1}: z_{2}$ electrolytes, where $z_{1}$ and $z_{2}$ are the valences of cations and anions. The electroneutrality in the bulk liquid phase requires that $n_{20}=n_{10} / \alpha$, where $n_{10}$ and $n_{20}$ being respectively the bulk concentrations of cations and anions and $\alpha=-z_{2} / z_{1}$. The porous particle is penetrable for ions and fluid and carries functional groups which may dissociate to generate counterions. We assume that the electric permittivity $\varepsilon$ takes the same value both inside and outside the polyelectrolyte particle. Spherical coordinates $(r, \theta, \varphi)$ is adopted to describe the liquid phase and the porous structure, and the cylindrical coordinates $(R, \Theta, Z)$ is applied to region of the cylindrical channel. The origin of the spherical coordinate is located at the center of the porous particle and the symmetric nature of the problem suggests that only half of the $(r, \theta)$ domain needs to be considered.

Based on Gauss's law, the electric potential and the space charge density $\rho$ are related by Poisson equation:

$$
\begin{gathered}
\nabla^{2} \phi=-\frac{\rho+\rho_{f i x}}{\varepsilon}=-\sum_{j=1}^{2} \frac{z_{j} e n_{j}}{\varepsilon}-\frac{\rho_{f i x}}{\varepsilon} \\
\nabla^{2} \phi=-\frac{\rho}{\varepsilon}=-\sum_{j=1}^{2} \frac{z_{j} e n_{j}}{\varepsilon}
\end{gathered}
$$

where $\nabla^{2}, n_{j}, z_{j}$, and $e$ are the Laplace operator, the number concentration of the ionic species $j$, the valence of the ionic species, and the elementary charge respectively. By setting the electric potential at zero where the ion concentration $n_{j}$ equals its bulk value $n_{j 0}$, we further assume that the distribution of ions obeys Boltzmann distribution, viz.,

$$
n_{j}=n_{j 0} \exp \left(-\frac{z_{j} e \phi}{k_{\mathrm{B}} T}\right)
$$

where $k_{\mathrm{B}}$ is the Boltzmann constant and $T$ is the absolute temperature. From (1)-(3), we obtain the following Poisson-Boltzmann equation, 


$$
\begin{gathered}
\nabla^{2} \phi=-\sum_{j=1}^{2} \frac{z_{j} e}{\varepsilon} n_{j 0} \exp \left(-\frac{z_{j} e \phi}{k_{B} T}\right)-\frac{\rho_{f i x}}{\varepsilon} \\
\nabla^{2} \phi=-\sum_{j=1}^{2} \frac{z_{j} e}{\varepsilon} n_{j 0} \exp \left(-\frac{z_{j} e \phi}{k_{B} T}\right)
\end{gathered}
$$

To simplify the treatment, subsequent discussions are based on scaled symbols, i.e., the governing equations are rewritten in dimensionless form. The following symbols are chosen for the characteristic variables: the radius of porous particle, $a$, the thermal electric potential per valence of cations, $\phi_{0}$ (defined as $k_{\mathrm{B}} T / z_{1} e$ ), and the bulk concentration of the cations, $n_{10}$. Corresponding dimensionless variables are listed as follows: $r^{*}=r / a, R^{*}=R / a, Z^{*}=Z / a, n_{j}{ }^{*}=n_{j} / n_{10}, \phi^{*}=$ $\phi / \phi_{0}$, and $Q_{f i x}=\rho_{f i x} a^{2} / \varepsilon \phi_{0}$. In terms of dimensionless variables, the electric potential can be expressed as follows:

$$
\begin{aligned}
& \nabla^{* 2} \phi^{*}=-\frac{(\kappa a)^{2}}{(1+\alpha)}\left[\exp \left(-\phi^{*}\right)-\exp \left(\alpha \phi^{*}\right)\right]-Q_{f i x} \\
& \nabla^{* 2} \phi^{*}=-\frac{(\kappa a)^{2}}{(1+\alpha)}\left[\exp \left(-\phi^{*}\right)-\exp \left(\alpha \phi^{*}\right)\right]
\end{aligned}
$$

where $\kappa^{-1}$ is the Debye screening length given by

$$
\kappa^{-1}=\sqrt{\varepsilon k_{\mathrm{B}} T / \sum_{j=1}^{2} n_{j 0}\left(e z_{j}\right)^{2}}
$$

The electric potential $\phi^{*}$ remains symmetry at the center of the porous particle owing to the spherical symmetry. The cylindrical wall $\left(R^{*}=R_{b}{ }^{*}=R_{b} / a\right)$ is assumed chargeless. In addition, the axial symmetry condition requires that $\partial \phi^{*} / \partial \theta=$ 0 at $\theta=0$. Infinitely far away from the sphere, the presence of the colloid should not be "felt", hence the electric field $\phi^{*}$ depending only on $R^{*}$, the radius of the micro-/nanochannel. In other words, $\phi^{*}=\phi_{\infty}{ }^{*}\left(R^{*}\right)$, where $\phi_{\infty}{ }^{*}\left(R^{*}\right)$ is the electric potential distribution within the pore without the presence of a particle. Note that in practice, however, one cannot assume that both the inlet and outlet are infinitely far away from the sphere. Instead, a finite value of $L / a$ is selected, with one-dimensional electric potential profile applied at both inlet $\left(Z^{*}=-L / a\right)$ and outlet $\left(Z^{*}=L / a\right)$. The solution is then considered to be convergent once the mobility thus calculated does not change with increasing value of $L / a$ anymore. According to our calculation experience, $L / a=6$ is long enough to justify the convergence. Hence $Z^{*}=L / a=6$ is chosen here to be the outlet location throughout our analysis.

The boundary conditions needed for the corresponding two-dimensional problem with the presence of a spherical particle are thus complete, and listed as follows:

$$
\begin{aligned}
\frac{\partial \phi^{*}}{\partial r^{*}}=0, & r^{*}=0, \\
\phi^{*}=0, & R^{*}=\frac{R_{b}}{a}, \\
\phi^{*}=\phi_{\infty}^{*}\left(R^{*}\right), & Z^{*}= \pm\left(\frac{L}{a}\right),
\end{aligned}
$$

$$
\frac{\partial \phi^{*}}{\partial \theta}=0, \quad \theta=0, \pi .
$$

A pseudo-spectral method based on Chebyshev polynomials is adopted here to solve the above equations associated with corresponding boundary conditions.

\section{B. Neutralization Fraction}

The driven-in space charges of counterions within the particle are regarded as if they are close enough to induce the physical condensation, hence neutralize the total amount of fixed charges to an effective value, $Q_{\text {eff }}$, defined as

$$
\begin{gathered}
Q_{\text {eff }}=\frac{\int_{0}^{1} \int_{0}^{\pi} \sum_{j=1}^{2} \frac{z_{j}}{z_{1}} n_{j}^{*} r^{* 2} \sin \theta d \theta d r}{\left.\frac{4}{3} \pi r^{* 3}\right|_{r^{*}=1}}+Q_{f i x} \\
=\frac{\int_{0}^{1} \int_{0}^{\pi} r^{* 2} \sin \theta\left[\exp \left(-\phi_{e}^{*}\right)-\exp \left(\alpha \phi_{e}^{*}\right)\right] d \theta d r^{*}}{\left.\frac{(1+\alpha)}{2 \pi(\kappa a)^{2}} \frac{4}{3} \pi r^{* 3}\right|_{r^{*}=1}}+Q_{f i x}
\end{gathered}
$$

The fraction of the fixed charges neutralized by these counterions is defined as a neutralization fraction:

$$
\theta=1-\frac{Q_{e f f}}{Q_{f i x}}
$$

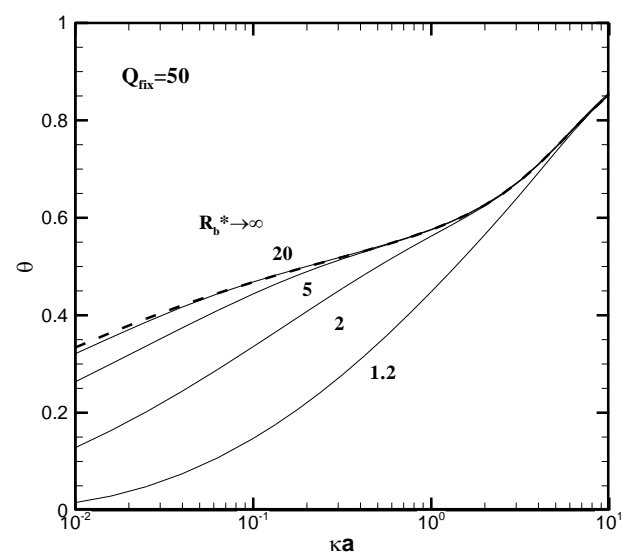

Fig. 2. Neutralization fraction of fixed charges, $\theta$, of a polyelectrolyte as a function of $\kappa a$ at different values of $R_{b}{ }^{*}$ with $Q_{f i x}=50$. Bold dashed: corresponding to results of a polyelectrolyte immersed in an unbounded electrolyte solution.

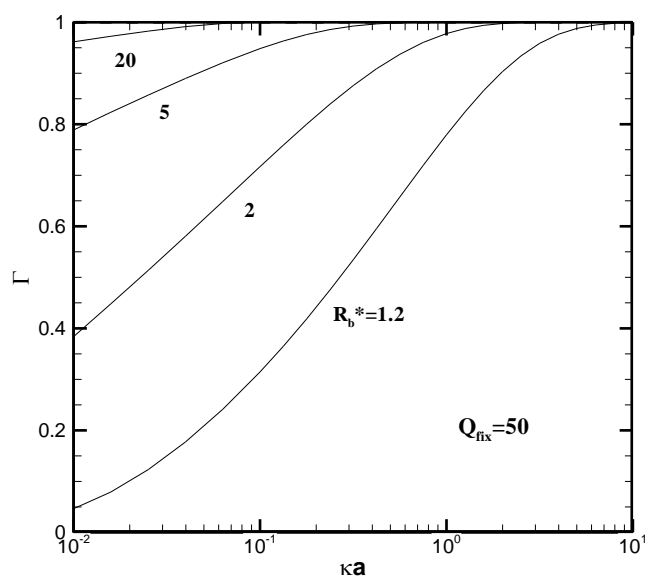

Fig. 3. Boundary factor of fixed charges, $\Gamma$, of a polyelectrolyte as a function of $\kappa a$ at different values of $R_{b}{ }^{*}$ with $Q_{f i x}=50$. 

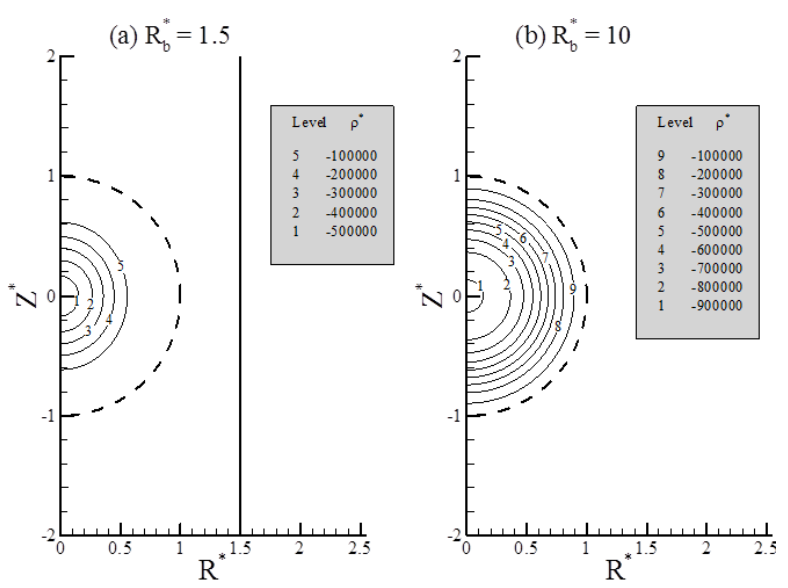

Fig. 4. Contour plots of the net amount of space charges, $\rho^{*}$, with $Q_{f i x}=50$, $\kappa a=0.01$ and (a) $R_{b}{ }^{*}=1.5$. (b) $R_{b}{ }^{*}=10$.

\section{RESULTS AND DISCUSSION}

A computer program developed by the authors employing a pseudo-spectral method is used to carry out all the numerical computations in this study. Standard mesh refinement test is conducted successfully to ensure the solution is grid-independent, a critical check for the convergence of a numerical solution in general. The accuracy and reliability of the present scheme is further verified by a direct comparison with the numerical results available in the literature for limiting cases, as reported by Hsu and Lee [11] where a porous sphere with fixed volumetric charge density $\rho_{f i x}$ immersed in an unbounded electrolyte solution. We compare our calculation results with Hsu's predictions for the corresponding contour of the electric potential, setting the pore-to-particle radius, measured by $R_{b}{ }^{*}$, to be close to infinity, or very large (Say, $R_{b}{ }^{*}=50$ ) to approach an unbounded case. The agreement is excellent. Less than $0.1 \%$ deviations are observed overall. We thus conclude our calculation results are both accurate and reliable.

Note that the dimensionless fixed charge density, $Q_{f i x}$ $\left(=\rho_{f i x} a^{2} / \varepsilon \phi_{0}\right)$ is function of the particle radius $a$. The actual range of $Q_{f i x}$ in practice can go over several orders of magnitude as a result, depending on the specific particle radius $a$ of interest. Nagasawa et al. [26] reported a Na-PVS polyelectrolyte system with $Q_{f i x}$ smaller than 100 . In reality, As far as the counterion condensation is concerned, $Q_{f i x}=50$ is high enough to represent the electrostatic behavior of a porous particle in general. Moreover, to conform to the practical application and emphasize the boundary confinement effect of a cylindrical pore, the radius of channel is confined to the same order of magnitude of the porous particle size.

We first investigate the impact on neutralization fraction of polyelectrolyte charges from the boundary confinement effect. Fig. 2 depicts the neutralization fraction $\theta$ as a function of $\kappa a$ at various pore-to-particle radii, $R_{b}{ }^{*}$. Note that the bold-faced dashed line represents the results of Hsu and Lee in 2012, and that $\kappa a$ represents the reciprocal of the double layer thickness in dimensionless form. Unlike a rigid particle with constant surface potential, the charged condition of a porous sphere is very sensitive to the concentration of the surrounding electrolyte ions in the solution. The higher the $\kappa a$ is, the more counterions are driven into the interior of the porous sphere, hence neutralizes the fixed charges there, leading to a larger fraction. Up to $80 \%$ amount of charges inside the porous sphere are neutralized when the electric double layer is thin $(\kappa a=10)$. Due to the reduction of the effective charges of the porous particle, the gradient of electrical potential near the particle surface decrease, hence depressing an interior induced electric field. This is referred to as the abovementioned shielding effect [6], [27], [28].

The degree of the neutralization fraction depends on $R_{b}{ }^{*}$ as well. The presence of the cylindrical channel "squeezes" the double layer surrounding the particle when it is thick hence affects the neutralization fraction by means of the electrostatic interaction. This phenomenon, however, tends to diminish when the double layer gets thinner, as shown in Fig. 2. Moreover, the smaller the radius of the cylindrical channel is, the lower the neutralization fraction of porous particle charges. To illustrate the influence of the radius of the cylindrical channel more specifically, we define the boundary factor $\Gamma$ as:

$$
\Gamma=\frac{\theta}{\theta_{\infty}}
$$

where $\theta_{\infty}$ represents the neutralization fraction of a porous sphere immersed in an unbounded electrolyte solution, which corresponds to the bold dashed line in Fig. 2. Rebuilded form Fig. 2, Fig. 3 depicting boundary factor $\Gamma$ as a function of $\kappa a$ at various pore-to-particle radii, $R_{b}{ }^{*}$. Fig. 3 shows that when the channel is narrow, say $\left(R_{b}{ }^{*}=1.2\right)$, the boundary affects up to $95 \%$ reduction of the neutralization fraction with $\kappa a=0.01$. On the other hand, when the channel is wide, say $\left(R_{b}{ }^{*}=20\right)$, the boundary affects the neutralization fraction weakly so that the boundary factor $\Gamma$ almost approaches 1 , which means the boundary effect is not significant.
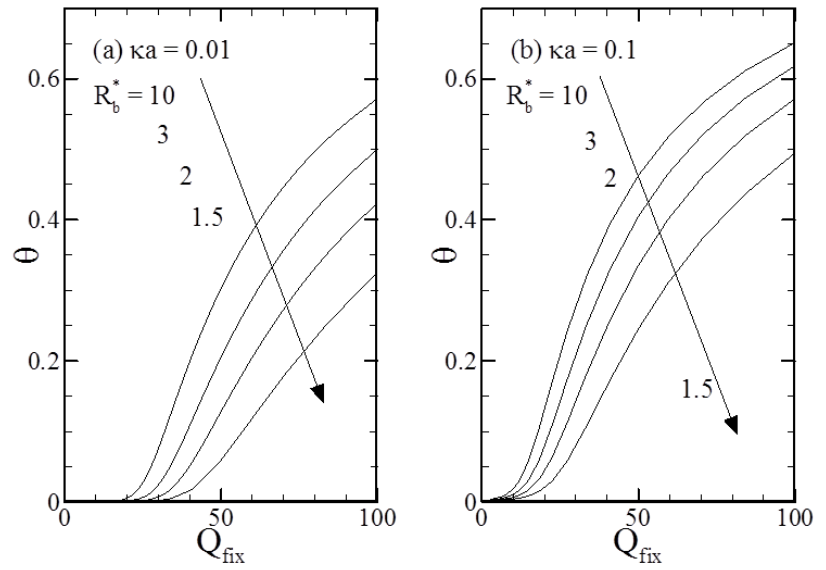

Fig. 5. Neutralization fraction of fixed charges, $\theta$, of a polyelectrolyte as a function of $Q_{f i x}$ at different values of $R_{b}{ }^{*}$ with (a) $\kappa a=0.01$. (b) $\kappa a=0.1$.

To explore the mechanism behind it, we present the contour plots of the net amount of space charges. As shown in Fig. 4, two representative values of pore-to-particle radii $\left(R_{b}{ }^{*}=1.5\right.$ and 10) are adopted to compare their space charges $\left(\rho^{*}\right)$ distribution. This is because the confining boundary effect squeezes not only the original double layer surrounding the particle but also the extra coions outside the double layer, hence increasing the effective charge density of the porous 
sphere instead. Indeed the electrostatic behavior in a nanostructure is fundamentally different from the one in a microstructure. Up to $50 \%$ reduction of dimensionless space charge density $\rho$ is observed for narrower channel $\left(R_{b}{ }^{*}=1.5\right)$ to wider one $\left(R_{b}{ }^{*}=10\right)$ at the center of the porous particle, which means less "effective" counterion inside the particle and hence leads to low neutralization fraction. This has direct impact in practical applications of biochemistry. For instance, by control of the neutralization of the total charges of macroions, people can manipulate the DNA condensation and RNA folding experimentally, which plays an important role in DNA vaccines and gene delivery.

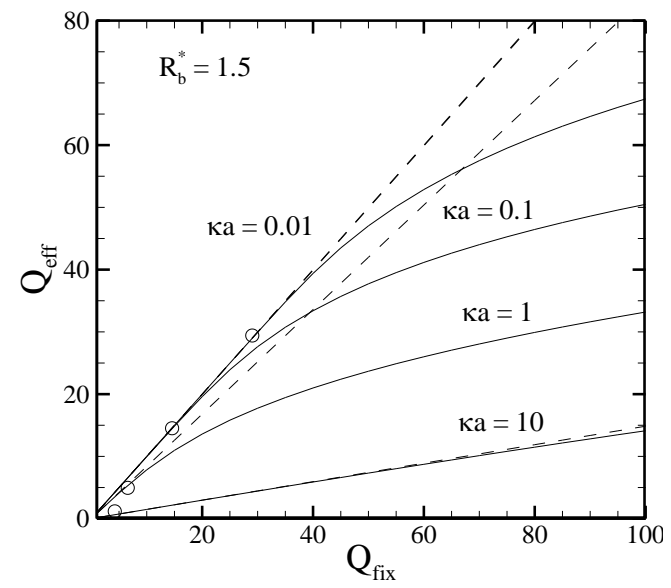

Fig. 6. Variation of the effective charge density, $Q_{\text {eff }}$, of the porous particle as a function of fixed charge density at various values of $\kappa a$ with $R_{b}{ }^{*}=1.5$. Dashed lines: results valid for low electric potential only taking no account of counterion condensation. Closed circles correspond to the approximate critical values of fixed charge density where the counterion condensation takes place.

To shed more light on the influence of the fixed charge density on particle's charge neutralization, we illustrate the neutralization fraction as a function of $Q_{f i x}$ at various levels of pore-to-particle radii $R_{b}{ }^{*}$. Considering the effect of the electric double layer thickness on $\theta$, we fixed the $\kappa a$ value for (a) $\kappa a=0.01$ and (b) $\kappa a=0.1$ respectively to represent thicker and thinner double layer thickness, as shown in Fig. 4. The neutralization fraction increases with increasing fixed charge density of porous particle monotonically. For smaller $Q_{f i x}$, the fixed charges of porous particle are too low to overcome strong Coulomb repulsive interaction between counterions, hence unable to attract more counterions and leading to $\theta$ approaches 0 . When $Q_{\text {fix }}$ goes higher, however, more counterions would be attracted into porous sphere and neutralize more fixed charges, thus increasing the value of $\theta$. It should be noted when $Q_{\text {fix }}$ gets very large, the neutralization fraction eventually approaches an upper limit value. This implies that the effect of counterions on the charge neutralization approaches a finite value with increasing $Q_{f i x}$.

Similar phenomenon is observed as well in Fig. 5, where narrower cylindrical pore leads to smaller neutralization fraction due to more coions squeezing into porous particle. Moreover, comparing Fig. 5(a) and (b), it can be found when the concentration of electrolyte solution increases (higher $k a$ ), the neutralization fraction raise accordingly regardless of pore-to-particle radii. This is because the higher the $\kappa a$ is, the more counterions would be, hence neutralizing more fixed charge on porous particle and leading to the shielding effect more significant.

As shown in Fig. 6, the effective charge density of a polyelectrolyte, $Q_{\text {eff, }}$, is depicted as a function of $Q_{f i x}$ at different double layer thickness. When $Q_{\text {fix }}$ is low, straight lines indicating linear relationship between the effective charge density and the fixed charge density is observed, corresponding to the results of the Debye-Huckel approximation reported by Hermans and Fujita [29], valid for the low electric potential situation. When the $Q_{\text {fix }}$ goes higher, however, the nonlinear behavior takes place leading to the counterion condensation eventually which reduces the effective charge density significantly. By observing the transition from linear behavior to non-linear behavior, we can pinpoint approximately a critical value of fixed charge density corresponding to the occurrence of counterion condensation. Note also that this critical value is a function of $\kappa a$ as well, a parameter that can be manipulated by the experimentalists. The corresponding case of wider cylindrical wall is shown as well for comparison purpose in Fig. 7. The neutralization of fixed charge density of porous particle is essentially not affected by the presence of the nearby cylindrical channel, if the double layer is very thin $(\kappa a=10)$. On the other hand, the thicker the double layer, indicated by small values of $\kappa a$, the more profound the boundary confinement effect in terms of effective charges reduction. This is understandable as the electrostatic impact from the presence of the micro/nanochannel is directly related to the degree of deformation of the double layer.

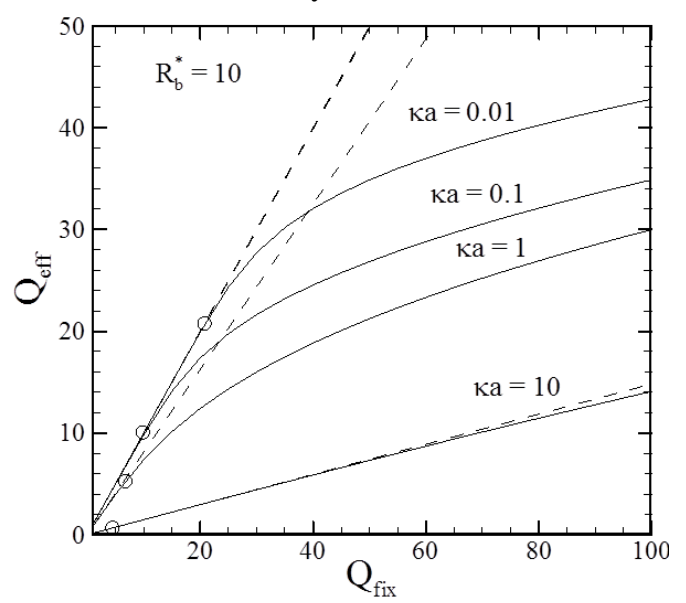

Fig. 7. Variation of the effective charge density, $Q_{e f f}$, of the porous particle as a function of fixed charge density at various values of $\kappa a$ with $R_{b}{ }^{*}=10$. Dashed lines: results valid for low electric potential only taking no account of counterion condensation. Closed circles correspond to the approximate critical values of fixed charge density where the counterion condensation takes place.

\section{CONCLUSION}

Excellent agreement with numerical data of the corresponding limiting case indicates the reliability of this analysis, as well as the success of using the charged porous sphere to model a polyelectrolyte system. In this study, we introduce a general model to evaluate the neutralization fraction quantitatively for a highly charged polyelectrolyte, even if severe counterion condensation has already takes place, such as a giant DNA in the extremely folded compact state. Additionally, the narrower pore-to-particle radii, the more extra coions swept inside the porous sphere due to the 
confining boundary effect, hence decreasing the neutralization fraction of the porous sphere. Indeed the electrostatic behavior in a nanostructure is fundamentally different from the one in a microstructure. This has direct impact in practical applications of biochemistry. For instance, by the control the neutralization of the total charges of macroions, people can manipulate the DNA condensation and RNA folding experimentally, which plays an important role in DNA vaccines and gene delivery.

\section{ACKNOWLEDGMENT}

This work is partially supported by the Ministry of Science and Technology, Republic of China.

\section{REFERENCES}

[1] R. M. Fuoss, "Polyelectrolytes," Science, vol. 108, pp. 545-550, 1948

[2] F. B. Surani and Y. Qiao, "Energy absorption of a polyacrylic acid partial sodium salt-modified nanoporous system," Journal of Materials Research, vol. 21, pp. 1327-1330, 2006.

[3] C. C. Lee, J. A. MacKay, J. M. Fréchet, and F. C. Szoka, "Designing dendrimers for biological applications," Nature Biotechnology, vol. 23 pp. 1517-1526, 2005.

[4] M. Shimomura and T. Sawadaishi, "Bottom-up strategy of materials fabrication: A new trend in nanotechnology of soft materials," Current Opinion in Colloid \& Interface Science, vol. 6, pp. 11-16, 2001.

[5] A. B. Lowe and C. L. McCormick, Polyelectrolytes and Polyzwitterions: Synthesis, Properties, and Applications, 2006.

[6] H. Ohshima and T. Kondo, "Electrophoretic mobility and Donnan potential of a large colloidal particle with a surface charge layer," Journal of Colloid and Interface Science, vol. 116, pp. 305-311, 1987.

[7] U. Böhme and U. Scheler, "Counterion condensation and effective charge of poly (styrenesulfonate)," Advances in Colloid and Interface Science, vol. 158, pp. 63-67, 2010.

[8] E. Nordmeier, Macromolecular Symposia, Wiley Online Library, pp. 21-37.

[9] V. L. Murthy and G. D. Rose, "Is counterion delocalization responsible for collapse in RNA folding?" Biochemistry, vol. 39, pp. 14365-14370, 2000.

[10] M. L. Bret and B. H. Zimm, "Distribution of counterions around a cylindrical polyelectrolyte and Manning's condensation theory," Biopolymers, vol. 23, pp. 287-312, 1984.

[11] H. P. Hsu and E. Lee, "Counterion condensation of a polyelectrolyte," Electrochemistry Communications, vol. 15, pp. 59-62, 2012.

[12] G. S. Manning, "Limiting laws and counterion condensation in polyelectrolyte solutions I. Colligative properties," The Journal of Chemical Physics, vol. 51, pp. 924-933, 1969.

[13] L. H. Yeh, J. P. Hsu, S. Qian, and S. Tseng, "Counterion condensation in pH-regulated polyelectrolytes," Electrochemistry Communications, vol. 19, pp. 97-100, 2012.

[14] G. Salieb-Beugelaar, K. Dorfman, A. Van den Berg, and J. Eijkel, "Electrophoretic separation of DNA in gels and nanostructures," Lab on a Chip, vol. 9, pp. 2508-2523, 2009.

[15] P. Dechadilok and W. M. Deen, "Electrostatic and electrokinetic effects on hindered diffusion in pores," Journal of Membrane Science, vol. 336, pp. 7-16, 2009.

[16] C. Ma and V. A. Bloomfield, "Gel electrophoresis measurement of counterion condensation on DNA," Biopolymers, vol. 35, pp. 211-216, 1995.

[17] W. Reisner et al., "Nanoconfinement-enhanced conformational response of single DNA molecules to changes in ionic environment," Physical Review Letters, vol. 99, p. 058302, 2007.

[18] C. Zhang, F. Zhang, J. A. van Kan, and J. R. Van der Maarel, "Effects of electrostatic screening on the conformation of single DNA molecules confined in a nanochannel," The Journal of Chemical Physics, vol. 128, p. 225109, 2008.
[19] Y. Kim et al., "Nanochannel confinement: DNA stretch approaching full contour length," Lab on a Chip, vol. 11, pp. 1721-1729, 2011.

[20] C. H. Huang, H. P. Hsu, and E. Lee, "Electrophoretic motion of a charged porous sphere within micro-and nanochannels," Physical Chemistry Chemical Physics, vol. 14, pp. 657-667, 2012.

[21] S. Tseng, C. Y. Lin, J. P. Hsu, and L. H. Yeh, "Electrophoresis of deformable polyelectrolytes in a nanofluidic channel," Langmuir, vol. 29, pp. 2446-2454, 2013.

[22] J. T. G. Overbeek, "Polyelectrolytes, past, present and future," Pure and Applied Chemistry, vol. 46, pp. 91-101, 1976.

[23] J. Mannion and H. Craighead, "Nanofluidic structures for single biomolecule fluorescent detection," Biopolymers, vol. 85, pp. 131-143, 2007.

[24] D. Bell and P. Dunnill, "The influence of precipitation reactor configuration on the centrifugal recovery of isoelectric soya protein precipitate," Biotechnology and Bioengineering, vol. 24, pp 2319-2336, 1982

[25] M. Y. Hussaini and T. A. Zang, "Spectral methods in fluid dynamics," Annual Review of Fluid Mechanics, vol. 19, pp. 339-367, 1987.

[26] M. Nagasawa, A. Soda, and I. Kagawa, "Electrophoresis of polyelectrolyte in salt solutions," Journal of Polymer Science, vol. 31, pp. 439-451, 1958

[27] N. Imai and K. Iwasa, "Theory of electrophoresis of polyelectrolytes," Israel Journal of Chemistry, vol. 11, pp. 223-233, 1973.

[28] Y. Y. He and E. Lee, "Electrophoresis in concentrated dispersions of charged porous spheres," Chemical Engineering Science, vol. 63, pp. 5719-5727, 2008

[29] J. Hermans and H. Fujita, "Electrophoresis of charged polymer molecules with partial free drainage," Proc. K. Ned. Akad. Wet., Ser. B: Phys. Sci., vol. 58, pp. 182-187, 1955.

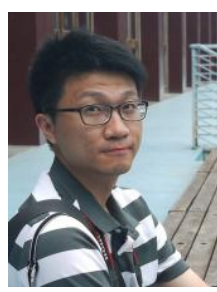

Cheng-Hsuan Huang received his $\mathrm{PhD}$ degree in 2012 from National Taiwan University under the supervision of Prof. Eric Lee. Currently, he works in Taiwan Semiconductor Manufacturing Company in Taiwan.

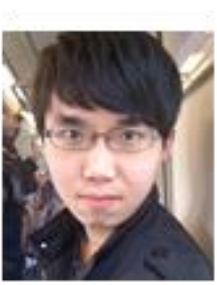

Yu-Fan Lee received his bachelor degree of chemical engineering from National Taiwan University in 2014. Now, he studies for a master degree under the supervision of Prof. Eric Lee, and works as a teaching assistant of Intermediate Numerical Methods Course in National Taiwan University.

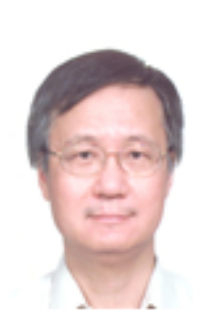

Eric Lee is a professor of the Department of Chemical Engineering at National Taiwan University, Taipei, Taiwan. His research interests include the fluid mechanics of systems containing polymers, such as polymer solutions, polymer melts, and other non-Newtonian fluids. The general electrokinetic behavior of colloidal particles of sub-micron or nano-scale dimensions is covered as well, focusing on the electrophoretic motion above all. The impact of air-water interface as well as particle migrations in gel or porous media is of particular interest in recent years. 